\title{
Shouting in a Political Echo Chamber
}

\section{Social media interactions with friends and political campaigns can lead to the emergence of polarized echo chambers of thought.}

\author{
By Katherine Wright
}

S ocial media has transformed political campaigns, allowing candidates to reach more people more quickly. But aggressive Facebook or Twitter marketing efforts might not be the way forward for those hoping to assert the most influence, according to results from Xin Wang of Beihang University, China, and colleagues [1]. Using a computational model, the team studied how psychological, social, and political processes can interact and lead to polarization through the emergence of political echo chambers. These echo chambers can be detrimental to campaigns, the team says, because they cause individuals to hold opinions that may be too extreme to persuade friends on opposite sides of the political spectrum.

In their study, the team considered a network model, where each node represented an individual whose backing was sought by two competing campaigns-red and blue. The individuals could interact with each other to discuss campaign messages and form new connections. They could also communicate directly with the two campaigns. The team tuned the degree of
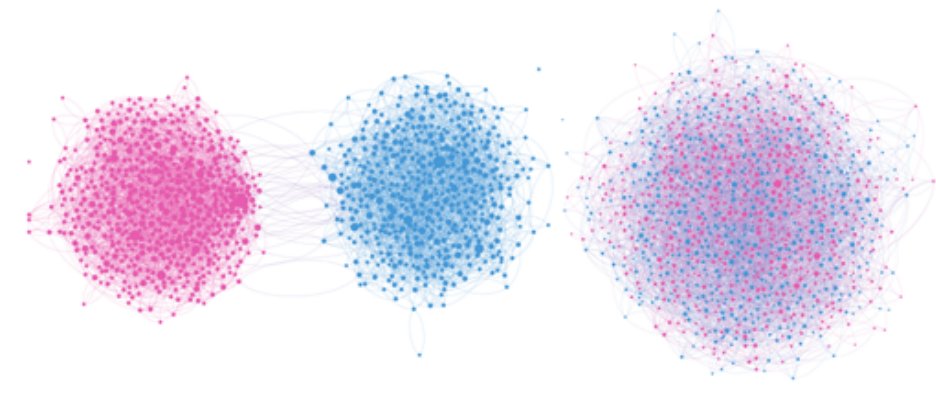

open-mindedness of individuals and analyzed how their opinions evolved over time.

They found three different regimes. When individuals were open to all opinions, the network formed a uniform blob of red and blue dots. When individuals were closed to opposing opinions, the network divided into many small, single-color groups. Finally, when individuals were open to opinions that were just a little different to their own, the network polarized into two groups with opposite political leanings. Increasing the strength of the political messaging, the team found that fewer and fewer individuals crossed from one group to another. That scenario may be unwanted, they say, as it becomes impossible to switch an individual's view.

Katherine Wright is a Senior Editor for Physics.

\section{REFERENCES}

1. X. Wang et al., "Public discourse and social network echo chambers driven by socio-cognitive biases," Phys. Rev. X 10, 041042 (2020).

Credit: X. Wang et al. [1] 